Fourth International Symposium on Physics of Fluids (ISPF4)

International Journal of Modern Physics: Conference Series

Vol. 19 (2012) 390-395

(C) World Scientific Publishing Company

DOI: $10.1142 /$ S2010194512008987

\title{
TURBULENCE IN THICK LAYERS
}

\author{
MICHAEL SHATS, HUA XIA AND DAVID BYRNE
}

Research School of Physics and Engineering, The Australian National University, Canberra, ACT, 0200, Australia Michael.Shats@anu.edu.au

\begin{abstract}
Properties of turbulence, such as the direction of the energy cascade depend on the flow dimensionality. Recent experimental results reveal new physics understanding of flows in thick layers. We show that the flow dimensionality can be characterized by the flow damping and that a comparison of the decay rate with that of a quasi-2D flow can be used as a measure of dimensionality of a turbulent flow in a layer. This dimensionality, however can be strongly affected if large scale coherent vortices are present in the flow. These vortices can imposed twodimensionality and reduce the damping rate. Finally we show that even in thick layers with unperturbed free surface, turbulent flow can be viewed as co-existing $2 \mathrm{D}$ top sub-layer and the bulk 3D flow.
\end{abstract}

Keywords: 2D and 3D turbulence; flows in fluid layers.

\section{Introduction}

Layered flows are those whose depths are much smaller than their horizontal extents. Planetary atmospheres and oceans belong to this class. The flow aspect ratio alone does not determine however whether turbulence in a layer would behave as $2 \mathrm{D}$ or $3 \mathrm{D}$. The principal difference between turbulence in two and three dimensions is the direction of the energy cascade ${ }^{1}$ : energy is transferred from smaller to larger eddies in $2 \mathrm{D}$, and from larger to smaller scales in 3D. The consequences of the energy flux are dramatic: the inverse energy cascade in 2D allows a flow self-organization. In 2D spectral energy can accumulate at large scales forming structures coherent across the system size. A remarkable progress has been achieved recently in understanding the energy transfer in turbulent flows in thick layers.

In this paper we review several new experimental results related to (1) turbulent flow dimensionality, (2) interaction between coherent vortices and turbulence in thick layers and (3) the spatial structure of turbulence. We study these issues in two main configurations: in single layers of a water-based electrolyte and in double layers where the top layer of electrolyte rests upon a heavier layer of electrically neutral fluid. In the latter case, turbulence is detached from the bottom and linear damping is substantially reduced. 


\section{Flow Dimensionality in a Single Layer}

Turbulence in layers is generated electromagnetically in a vertical spatially varying magnetic field crossed with the electric current flowing across the layer, as discussed in Refs. 2 and 3. Magnetic dipoles placed under the fluid cell generate small vortices (8-9 $\mathrm{mm}$ in diameter) which then interact with each other forming complex turbulent motion. Horizontal fluid motion is studied using 2D PIV, while vertical motions are studied using the defocusing PIV technique combined with the particle streak imaging in the vertical plane ${ }^{4}$.

In the absence of $3 \mathrm{D}$ motions, the flow in a layer is damped due to the bottom friction due to the molecular viscosity. A decay of horizontal velocity $V_{x, y}(z, t)$ in a quasi-2D flow due to the bottom friction is given by the decay rate of the energy of $\alpha_{L}=v \pi^{2} / 2 h^{2}$. Here $v$ is the kinematic viscosity and $h$ is the layer thickness. The onset of $3 \mathrm{D}$ turbulent eddies leads to a vertical flux of horizontal momentum and faster dissipation of the flow. Such a flux is related to the mean vertical velocity gradient:

$$
\left\langle\tilde{V}_{x, y} \tilde{V}_{z}\right\rangle=-K\left(\partial V_{x, y} / \partial z\right)
$$

where $K$ is the eddy (turbulent) viscosity coefficient.
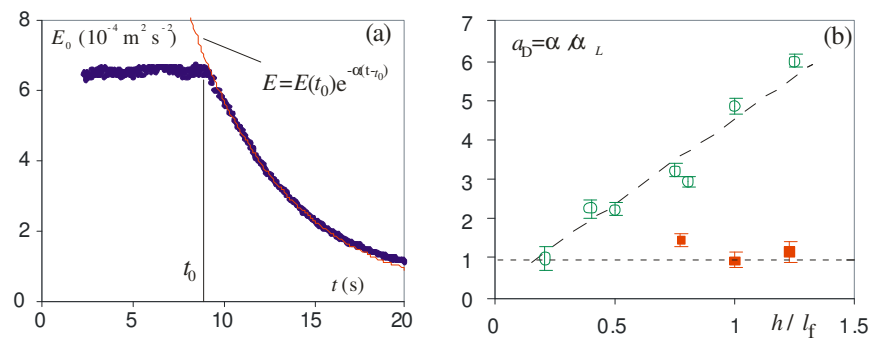

Fig. 1 (a) Decay of the flow energy in layer $10 \mathrm{~mm}$ thick. (b) Energy damping rate normalized by the viscous quasi-2D damping rate, $a_{D}=\alpha / \alpha_{L}$ as a function of the thickness layer normalized by the forcing scale, $h / l_{f}$. Open circles correspond to single layers, solid circles show data obtained in the double layer configurations.

Figure 1(a) shows experimentally measured decay of the flow energy together with the exponential fit. Fig. 1(b) shows the decay rate normalized by the viscous quasi-2D damping rate, $a_{D}=\alpha / \alpha_{L}$, as a function of the normalized layer thickness. For the thinnest layer, the measured decay rate coincides with the quasi-2D estimate, while for thicker layers, this anomaly coefficient increases linearly with the thickness. However in the double layer configuration, the anomaly coefficient $a_{D}$ remains low even in much thicker layers.

This result can be understood in the frame of the eddy viscosity concept. As the layer thickness is increased, 3D motions become more and more visible, Fig. 2(a-c). RMS of horizontal velocities increases roughly proportional to the layer thickness, Fig. 2(d). The eddy viscosity coefficient can be estimated from experimental data as $K \approx\left\langle\tilde{V}_{x, y}\right\rangle\left\langle\tilde{V}_{z}\right\rangle\left\langle\partial \tilde{V}_{x, y} / \partial z\right\rangle^{-1}$. Then the damping rate is estimated using contributions 
from both molecular and eddy viscosity, $\alpha_{t} \approx(v+K) \pi^{2} / 2 h^{2}$. The ratio of thus computed damping rate to the linear damping $\alpha_{L}$ is shown in Fig. 2(e). One can see that the damping anomaly coefficients estimated from the eddy viscosity model, Fig. 2, and from the direct measurements of damping, Fig. 1, agree pretty well.
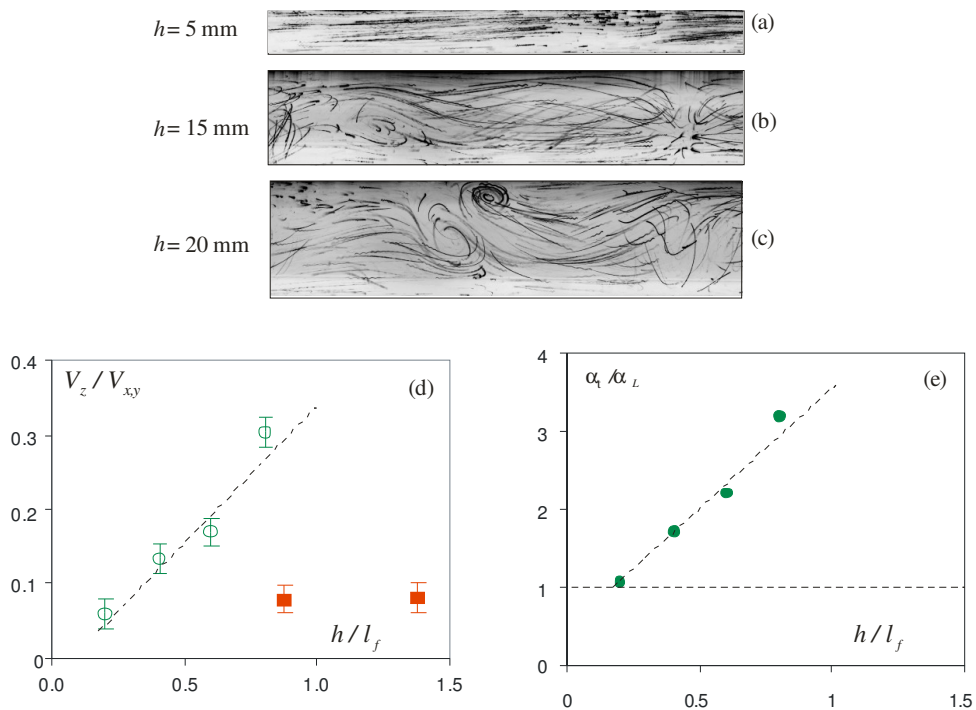

Fig. 2 (a-c) Particle streak photos in single layers of different thicknesses. (d) RMS of the vertical velocity fluctuations normalized by the RMS of horizontal velocities. (e) The anomaly coefficient estimated using Equation (1), as $a_{D}=\alpha_{t} / \alpha_{L}$.

This result ${ }^{4}$ suggests that the anomaly coefficient, which characterizes the deviation of damping from the one derived using a quasi-2D model, can be used as a practical measure of the flow dimensionality.

\section{Two-dimensionality Imposed by Large coherent Flow}

Now we investigate how large-scale coherent flows affect the dimensionality of turbulence in thick layers. Some of these results were published in Ref. 5. When turbulence is generated in a single layer, the flow is subject to substantial bottom drag. Such a drag can dampen the inverse energy cascade. This is shown in Fig. 3(a-b). The $3^{\text {rd }}$-order structure function of the velocity fluctuations is very small in turbulence, and the spectrum is flatter than the $k^{-5 / 3}$ Kolmogorov-Kraichnan scaling.

When the large scale coherent vortex is imposed externally on top of turbulence, the bottom drag is strongly reduced, such that the third-order structure function $S_{3}$ increases and becomes a positive linear function of the separation distance, as seen in Fig. 3(b). This is indicative of a strong inverse energy cascade in 2D turbulence. This result is very similar to that in a double layer configuration, Figs. 3(c-d), where the large-scale coherent vortex develops as a result of self-organization of turbulence in the process of spectral condensation. 

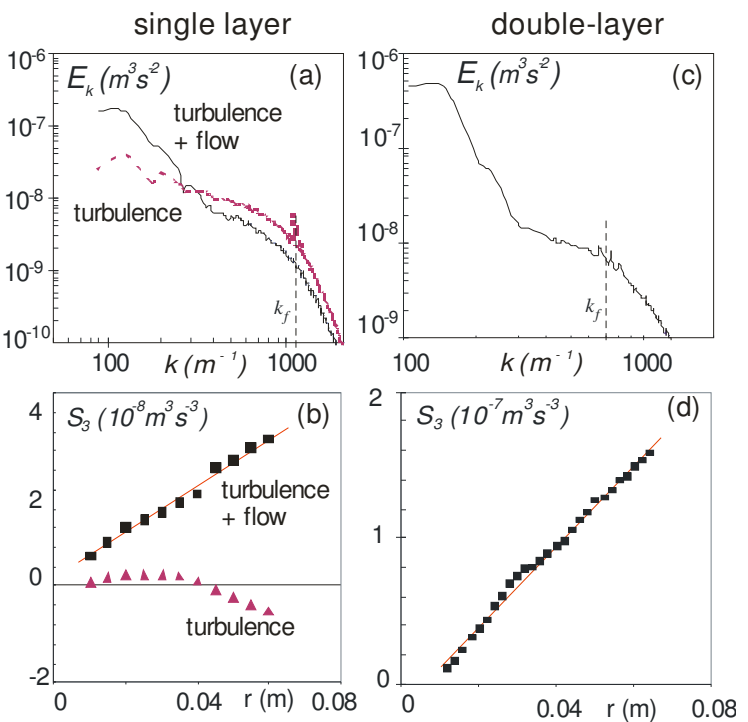

Fig. 3 Effect of the large-scale coherent vortex on (a) turbulence spectrum and on (b) third-order structure function $S_{3}$ in a single layer $\left(h=10 \mathrm{~mm}, l_{f}=8 \mathrm{~mm}\right.$ ). In a double layer (top layer thickness $7 \mathrm{~mm}, l_{f}=9 \mathrm{~mm}$ ), where large coherent vortex develops as a result of spectral condensation, the (c) spectrum and (d) $S_{3}$ are similar to those with externally imposed flow.
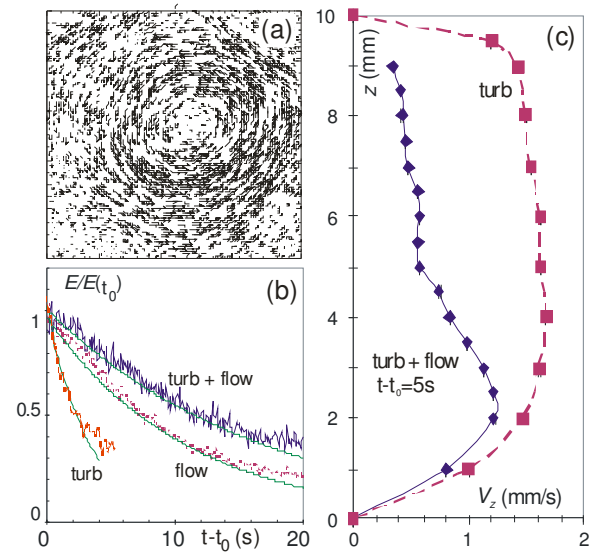

Fig. 4 (a) Velocity field of the coherent vortex externally imposed on turbulence (b) Time evolution of the total kinetic energy during the flow decay. (c) Vertical profiles of the RMS of the vertical velocity fluctuations in a single layer in turbulence (squares) and with the coherent vortex imposed on turbulence (diamonds).

The above results can be explained as follows. The imposition of the large-scale coherent flow on turbulence leads to the shear suppression of the vertical eddies. This in turn leads to the reduction in the eddy viscosity, to the reduction in the bottom drag, and eventually to the enhancement of the (otherwise dampened) inverse energy flux. How the same scenario is realized in the double layer configuration will be clarified in the next section. 


\section{Spatial Structure of Turbulence in Thick Layers}

Now we consider vertical velocity profiles in a single layer configuration. Figure 5 shows vertical profiles of vertical (a) and horizontal (b) velocities. RMS vertical velocities $<V_{\mathrm{z}}$ $>$ are low in the top sublayer, $2 \mathrm{~mm}$ below the free surface, as well as in the bottom boundary sublayer. In the bulk of the flow $(2-8 \mathrm{~mm})<V_{\mathrm{z}}>$ is high, being only a factor of two lower than horizontal velocities $\left\langle V_{\mathrm{x}, \mathrm{y}}\right\rangle$. $\left\langle V_{\mathrm{x}, \mathrm{y}}\right\rangle$ shows a maximum at $h=(3-4)$ $\mathrm{mm}$, which is indicative of the competition between the forcing and the bottom drag. In the top sublayer, $h_{1}=(8-10) \mathrm{mm}$, turbulence is expected to behave as quasi-2D due to the lower vertical velocities and the absence of vertical gradients of the horizontal velocities.
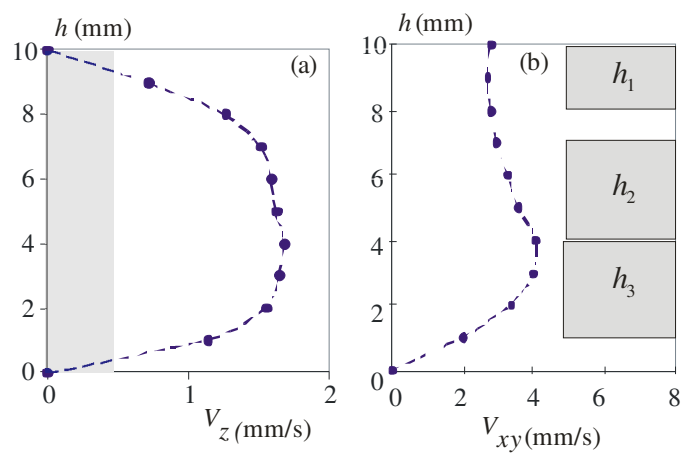

Fig. 5 Vertical profiles of (a) vertical, $V_{z}$, and (b) horizontal, $V_{x ; y}$, velocities. A grey box in (a) indicates the sensitivity of the defocusing PIV technique. Grey boxes in (b) show the regions within the layer visualized using laser light.

To test if the nature of the turbulent energy transfer changes between the top sublayer, the bulk flow and the bottom layer, we perform PIV measurements of the horizontal velocities by illuminating three different ranges of heights: $h_{1}=(8-10) \mathrm{mm}, h_{2}=(4-7)$ $\mathrm{mm}$, and $h_{2}=(1-4) \mathrm{mm}$.

Figures 6 (a) and (b) show the kinetic energy spectrum and the third-order structure function as a function of the separation distance measured in the top sublayer $h_{1}$. At $k<k_{f}$ the spectrum scales close to $k^{-5 / 3}$, while $S_{3}$ is positive at $r>l_{f}$ and is a linear function of $r$. This is in agreement with the expectation of the quasi-2D turbulence in the top sublayer.

In the bulk flow $h_{2}$, which is dominated by $3 \mathrm{D}$ motions, the spectrum is still close to $k^{-5 / 3}$, though it flattens at low wave numbers. Consistently with this, the range of scales for which $S_{3}$ is positive and linear is reduced to about $\mathrm{r} \approx 40 \mathrm{~mm}$.

In the bottom sublayer $h_{3}$, Figs. 6(c) and (d), the flow is subject to even stronger damping. As a result, the spectrum is much flatter than $k^{-5 / 3} \cdot S_{3}$ is positive for a narrow range of scales, giving a hint of same trend as in the bulk and the top sublayers.

The above results suggest that despite the presence of substantial 3D motion in a thick $\left(h / l_{f}=1.28\right)$ single layer, statistics of the horizontal velocity fluctuations remain consistent with that of quasi-2D turbulence and supports the inverse energy cascade. 

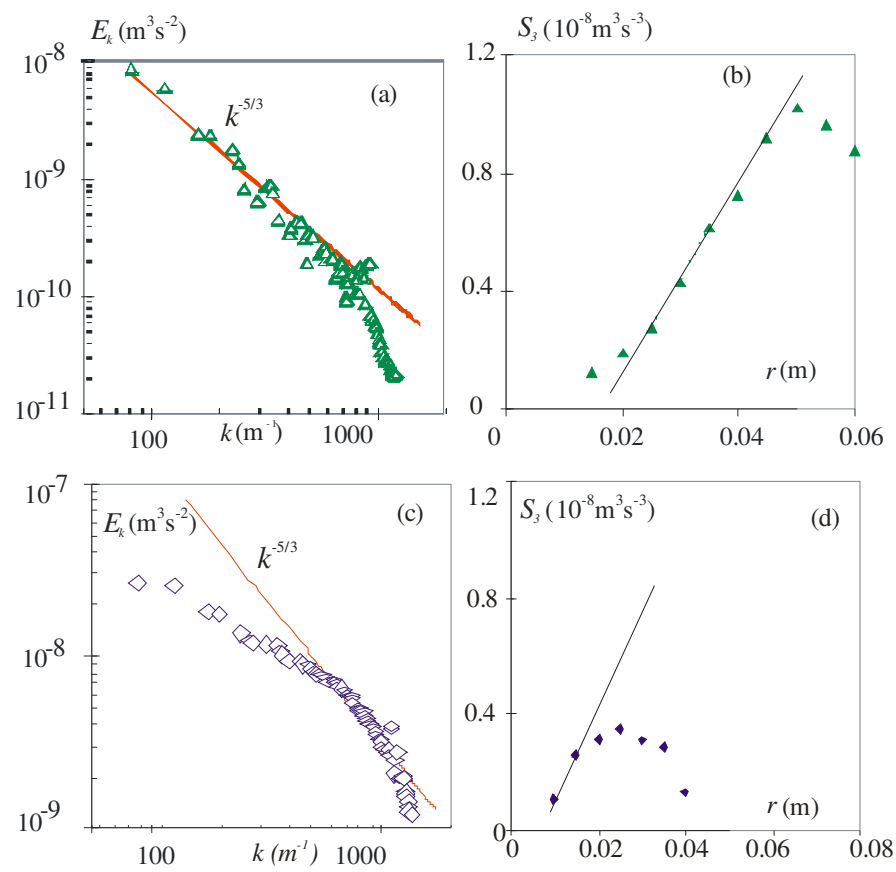

Fig. 6 (a, c) Wave-number spectra and (b, d) the third order structure functions $S_{3}$ measured in (a, b) the surface sublayer $\left(h_{1}=(8-10) \mathrm{mm}\right),(\mathrm{c}, \mathrm{d})$ in the bottom sublayer regions, $h_{3}=(1-4) \mathrm{mm}$.

\section{Conclusions}

Thus we conclude that flows in thick layers of fluids with an unperturbed free surface can be viewed as two interacting sublayers. The top layer is quasi-2D; it supports the inverse energy cascade. In a bounded domain at low damping, the inverse cascade leads to spectral condensation of turbulence. The bottom sublayer is dominated by $3 \mathrm{D}$ motions which are responsible for the onset of the eddy viscosity. A planar coherent flow (spectral condensate) developing in the top layer can reduce the bottom layer thickness through shearing of the 3D eddies. The thickness of the two sublayers thus depends on the competition between the vertical shear and the 3D motions due to the forcing. In the two layer configuration, the spectral condensate formed in the top sublayer can take over almost the entire layer thickness.

\section{Reference}

1. R.H. Kraichnan, Phys. Fluids, 10, 1417 (1967).

2. H. Xia, M. Shats and G. Falkovich, Phys. Fluids, 21, 125101 (2009).

3. H. Xia and M. Shats, Structure formation in spectrally condensed turbulence. This conference.

4. M. Shats, D. Byrne, H. Xia, Phys. Rev. Lett. 105, 264501 (2010).

5. H. Xia, D. Byrne, G. Falkovich and M. Shats, Nature Phys. 7, 321 (2011).

6. D. Byrne, H. Xia and M. Shats, Robust inverse energy cascade and turbulence structure in three-dimensional layers, Phys. Fluids, 23 (2011) in press. 